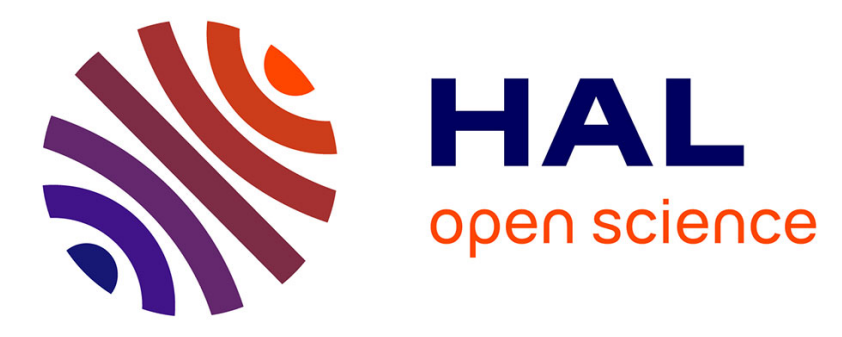

\title{
Towards product health monitoring throughout its life-cycle
}

Nicolas Krommenacker, Patrick Charpentier, Rodolfo Pena

\section{To cite this version:}

Nicolas Krommenacker, Patrick Charpentier, Rodolfo Pena. Towards product health monitoring throughout its life-cycle. 11th IFAC Workshop on Intelligent Manufacturing System, IMS'2013, May 2013, Sao Paulo, Brazil. pp.169-173, 10.3182/20130522-3-BR-4036.00090 . hal-00835483

\section{HAL Id: hal-00835483 https://hal.science/hal-00835483}

Submitted on 18 Jun 2013

HAL is a multi-disciplinary open access archive for the deposit and dissemination of scientific research documents, whether they are published or not. The documents may come from teaching and research institutions in France or abroad, or from public or private research centers.
L'archive ouverte pluridisciplinaire HAL, est destinée au dépôt et à la diffusion de documents scientifiques de niveau recherche, publiés ou non, émanant des établissements d'enseignement et de recherche français ou étrangers, des laboratoires publics ou privés. 


\title{
Towards Product Health Monitoring throughout its life-cycle
}

\author{
Nicolas KROMMENACKER*, Patrick CHARPENTIER*, Rodolfo PEÑA* \\ *Centre de Recherche en Automatique de Nancy (CRAN)- CNRS UMR7039 \\ Université de Lorraine, BP 239, 54506 Vandouvre, France \\ (e-mail: firstname.lastname@univ-lorraine.fr)
}

\begin{abstract}
Product Health Monitoring (PHM) is the process of monitoring usage conditions of the product. Like Structural Health Monitoring systems, they are traditionally deployed during the MiddleOf-Life (MOL) phase of the product's life-cycle. The aim of this paper is to describe the concept of PHM throughout its life-cycle. Advances in Nanotechnology and wireless nano sensors networks allow envisaging their integration into many products and systems. From this observation, we propose to describe a system to monitor dimensional features of a product. The monitoring system uses selforganizing communication network principles. Thereby the product can perform its continuous health monitoring, regardless its physical transformations. Self-measurement system requires no specific sensors and is based on two techniques: neighborhood discovering and distance-ranging.
\end{abstract}

Keywords: Health Monitoring, Intelligent Product, Wireless Nano Sensor Networks

\section{INTRODUCTION}

Product Health Monitoring (PHM) is a field of interest of researchers since the last decades. This concept is defined like the observation of the usage conditions of a product along to its life-cycle, and the environmental degradations it undergoes. The latter includes physical or electrical degradation (e.g. corrosion, radiation and electrical stresses) and changes in life-cycle environment (e.g. ambient humidity and temperature, usage duration and frequency, etc.) (Vichare et al. (2004)). Like Diagnostic or Prognostic systems, performance criteria and appropriate tools must be defined through observation (continuous or periodic measurement of physical parameters) and interpretation of measured data (product's degradation assessment) (Mishra et al. (2002)).

The application of PHM is well established for the assessment of systems where electronic is integral to the functionality, like notebook computer. The hardware incorporates local sensors and the system can be easily monitored from the collection and analysis of operating parameters. Hence, the challenge is to identify relevant parameters that should be measured and develop monitoring strategies to prevent failure or performance degradation (Niu et al. (2011)).

Otherwise, it requires adding capabilities to the product such as signal acquisition, processing, analysis and transmission. Micro Electromechanical Systems (MEMS) plays here a significant role in the development of such PHM systems. Driven by Moore and more than Moore's laws, advanced sensors today enable to develop efficient data acquisition systems. The networked sensors (using mainly wireless technologies) also allow health monitoring for large scale products. The birth of the Structural Health Monitoring
(SHM) community in the final decade of last century and the growing amount of research investigation for army systems, civil aircraft or civil infrastructures, illustrate the interest of such systems (Balageas et al. (2010)). However, these systems are traditionally used during the Middle-Of-Life (MOL) phase of the product's life-cycle. They are specifically tailored and adapted to the product to monitor, but are not integrated to the product itself.

Nanotechnologies are expected to be one of the most important technological breakthroughs. The developments of processors, sensors or actuators at the nano-scale (Nano Electromechanical Systems), allow envisaging the integration into many products and systems. Advances in miniaturisation lead also to design nano-networks, consisting of individual stations communicating over a wireless medium using nanotransceivers and nano-antennas. Hence, it could be possible to monitor a product throughout its life-cycle, from its Beginning-Of-life (BOL) to the End-Of-Life (EOL). This opportunity opens a range of new application of PHM systems, like monitoring specific features of the product from production to its recycling phase. In this paper, we propose to highlight how nano-sensors networks integrated into products could offer these new functionalities. We focus particularly on concept of intelligent products with self-measurement capabilities based on Wireless Nano-Sensors Networks (WNSNs).

The remainder of this paper is organized as follows: we present PHM systems according to the Intelligent Product concepts. Then an overview of technologies enabling PHM throughout life-cycle will be given in Section 2. The selfmeasurement concept of product's dimensions will be described in Section 3, and developments discussed in Section 4. In Section 5, concluding remarks are given. 


\section{PRODUCT HEALTH MONITORING SYSTEMS}

\subsection{Intelligent products and PHM systems}

By augmenting the product with computational and communication abilities, perception and control capabilities, the concept of Intelligent Products is emerged (Främling and McFarlane (2009)). Several definitions of this concept have been found in the literature mainly due to no standard definition of intelligence (Legg et al. (2007)). Although there is no single standard definition, Meyer et al. (2009) proposed a classification of Intelligent Products depending on the level of intelligence, the location of the intelligence and the aggregation level of intelligence. This three-dimensional classification model (Fig. 1.) can be used to classify the Intelligent Products according to the discussed applications. For instance a notebook computer including PHM system can be described like an Intelligent Product with:

- A level of Intelligence allowing it to manage its own information from sensors (e.g. temperature, luminosity), notify a problem to the owner or manufacturer (e.g. lowbattery level indication, software/hardware bug reports) and take some decisions (e.g. adjust fan speed or screen brightness).

- Location of Intelligence at the physical product itself due to its intrinsic computational power, storing capacities and network connectivity.

- Intelligence distributed into components which can be removed or replaced without impacting the level of intelligence.

Hence, the concept of PHM throughout life-cycle is more consistent with the definition given by Ventä (2007). Ventä refers to systems that:

- Continuously monitor their status and environment,

- React and adapt to environmental and operational conditions,

- Maintain optimal performance in variable circumstances, also in exceptional cases,

- Actively communicate with the user, environment or with other products and systems.

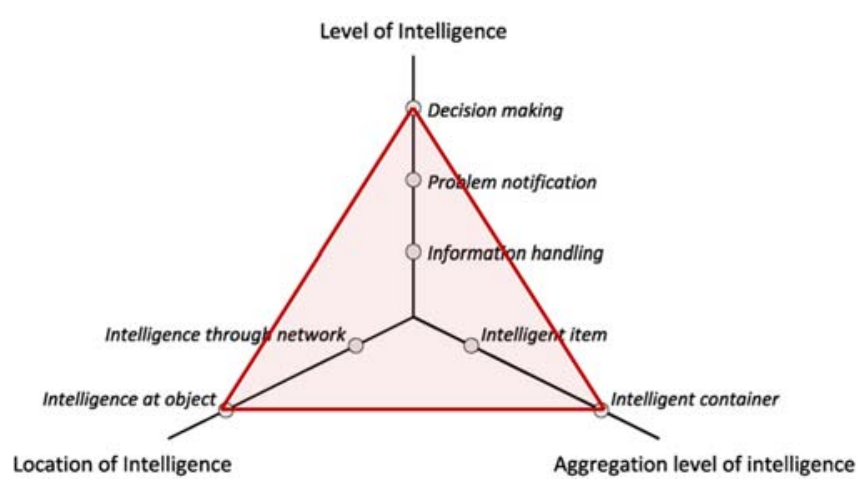

Fig. 1. PHM systems and intelligence levels.
A product integrating its own Health Monitoring system could be defined as an Intelligent Product characterized by the highest point on each axis of the model (Fig. 1.). While this vision involves embedding sufficient microelectronics, the trend of MEMS and NEMS technologies and their integration of the material level enable to consider new functionalities of health monitoring for one product.

\subsection{Technologies enabling PHM}

Nanotechnology offers the opportunity to produce mechanical, electromechanical, or electrochemical devices with the nano-dimensional scale. This emerging technology has enabled the fabrication of nanoscale circuitries (e.g. nano-processor, nano-memory, nano-transceiver, nanosensors) and nano-devices to perform sensing and actuation functions (Fig. 2.). Three approaches in implementation are developed (Akyildiz et al. (2010)). The first and more classical approach, commonly called top-down approach, uses the same techniques as used in MEMS, but with smallsize constraints approaching a nanometer. Advanced nanofabrication processes as lithography, deposition and etching are here employed. An opposite approach is to manipulate individually the material at the atomic or molecular level (mainly via chemical reactions) to produce a desired component by precise assembly, such as carbon nanotubes, nanowires or nanoparticles (bottom-up approach). Finally, the third approach is the bio-hybrid approach, where biological components (e.g. cells) are building blocks of integrated nano-devices.

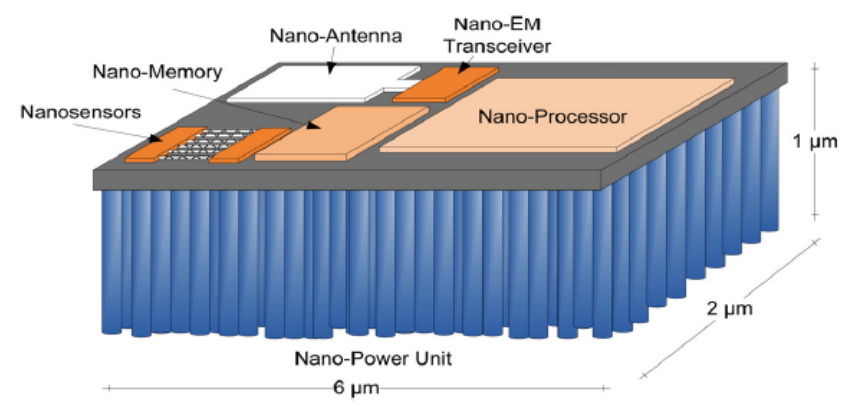

Fig. 2. Schematic view of a nano-device with nanoscale circuitries.

The possibility of building components at the nanoscale revolutionized the way we think about systems. In the case of Health Monitoring system where sensors are core elements, the development of nano-sensors with nano-communication capabilities enables to extend the Wireless Sensor Networks paradigm to the Wireless Nano-Sensor Networks (WNSNs). They are collections of nano-nodes including the sensing, interfacing and processing functions. Even if they are still at a very early stage, there exist several research works in the literature for the realization of nano-scale sensor networks. For instance, Koksal et al. (2010) and Atakan et al. (2009) focus on nanoscale communication systems equipped with carbon nanotube-based receivers and transmitters. Kocaoglu et al. (2012) investigate a novel energy coding scheme to 
minimize energy consumption for transmission in nanosensor networks. Communication characteristics of a molecular nano networks are also studied in Parcerisa et al. (2009) and Moore et al. (2010).

The emergence of nano-sensor networks and their integration in the materials is consistent with augmented materials concept where a network of distributed sensors and actuators is embedded into materials in order to give it self-* abilities (i.e., self-identification, self-testing, self-healing, etc.) (Delaney (2008)). Also named smart materials, the material would know itself, and its current status. The notion of composition which is the interaction of several smart materials to be considered as a unique one is very attractive too. This concept of self-organization is challenging and opens a range of new applications.

\subsection{PHM and self-organization}

Self-organization applied to wireless communication networks is not a new concept (Robertazzi et al. (1986)). Largely deployed in wireless sensor networks today, it provides several functions like sharing processing resources, maintaining communication capacity and minimizing energy consumption. Adaptation of these techniques to wireless nano sensor networks offers a new way to envisage the monitoring of products. Indeed a smart product embedding its own PHM system based on previous technologies can undergo several transformations (e.g. cutting, assembling) along to its lifecycle. Self-organization techniques allow to each parts to react and maintain continuous health monitoring.

To illustrate it, section 3 describes the concept of intelligent product able to monitor its own dimensions regardless transformations.

\section{CONCEPT OF SELF-MEASUREMENT SYSTEMS}

Based on specific sensing technologies, communication status between the smart product itself and the end-users or manufacturers is the most cited scenario of ubiquitous computing. In order to illustrate interest of PHM systems throughout product life cycle, we focus here the ability of each intelligent product to differentiate themselves even after evolution of life-cycle phases. The main idea is to use dimensional features of products to do this. Based on wireless nano sensor network, the intelligent product can determined its own dimensions and then communicate information. If physical transformation is operated on the product for example during the production or recycling phase, each component is able to establish again its new properties. Figure 3 illustrates this concept of self-measurement systems.
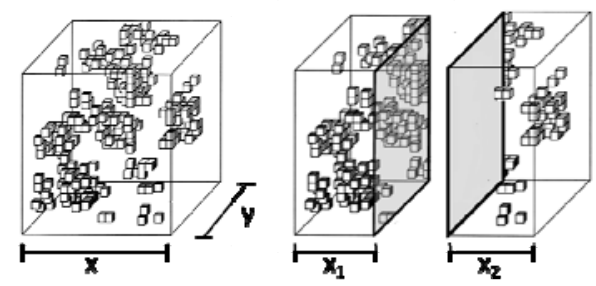

Fig. 3. Self-measurement systems.
The self-measurement strategy is described with two steps. From nano devices deployed inside the product, the first one consists in identifying nano nodes which are situated on outlines of the product (Figure 4a to $4 \mathrm{~b}$ ). Algorithms based on neighborhood density estimation will be used. Then the distance estimation based on localization techniques between these border nano-nodes will provide dimensional information about the product (Figure $4 \mathrm{~b}$ to $4 \mathrm{c}$ ).

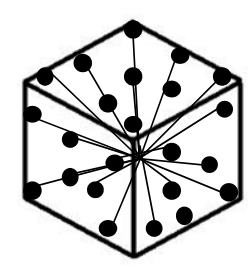

(a)

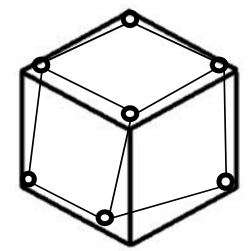

(b)

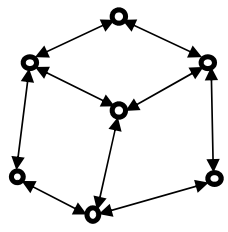

(c)
Fig. 4. Steps of self-measurement strategy.

To do this, we consider the product has been endowed with the necessary nano-devices. These nano-nodes have autonomous computing capacity and a wireless communication channel. The proposed strategy uses only communication between nodes and no specific sensors are needed. The implementation of the nano-network in the product must follow these constraints:

- The physical distribution of nano nodes is an important part of the PHM system's performance. The deployment pattern related to the nature and shape of the product must be uniform in the material. This hypothesis is necessary to ensure that nano nodes are distributed within all parts of the product, and allow to determine dimensions close to real size of the product.

- The radio propagation is a spherical model and the transmission range is identical for all nodes. It is dependent of the type of material and its homogeneity. All nano nodes have the same transmission characteristics whatever their position in the product in order to estimate the neighborhood density.

- The localization techniques used based on ranging are suitable and not disturbed by materials.

Under these conditions the proposed concept confers selfmeasurement capabilities to the product. This system presents several advantages like the communication capabilities in order to communicate their dimensions with either end-users or other products. Moreover the autonomy to perform the measurements itself enables to each part obtained after transformation to recalculate its dimensions, and this, whatever its life-cycle. 


\section{CONCEPT DEVELOPMENT}

\subsection{Neighborhood density estimation}

To determinate the border nano nodes within the wireless nano sensor networks, a neighborhood discovery technique is used. For each node, this process allows identifying his neighbors. Then, under implementation assumptions made in section 3, the estimation of the neighborhood density allows to determinate nodes with the lowest quantity of neighbors, i.e. the border nodes. Figure 5 shows an example of the quantity of neighbors for each node distributed on a square grid. With a coverage radius $r$ equals to 1 (represented with gray area on the figure and identical for all nano nodes), all nodes represented by a triangle form have 4 neighbors. There are situated within the product. The border nodes represented by circle form possess the minimum number of neighbors (equal to 2 in this case). Distance estimation between these nodes will be permit to obtain dimensions of the product.

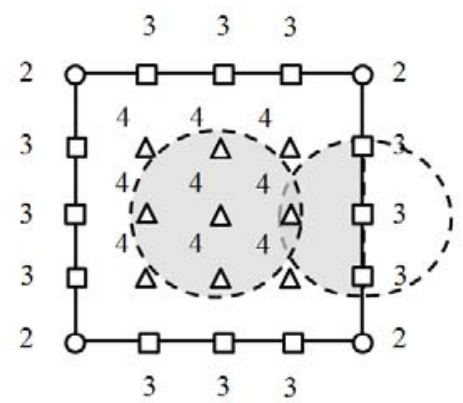

Fig. 5. Neighborhood density estimation

This technique is applicable to other geometric figures, with the same assumptions. However, if the particles are randomly distributed in the product, it is important to ensure that the neighborhood density techniques permits to find border nodes. The problem has been formulated for simple 2D figures and could be easily extend to $3 \mathrm{D}$, with $2 \mathrm{D}$ layers representation. Problem formulation for a square form is the following:

Considering a square of side $A$, and a number $N$ of nano nodes deployed randomly over the surface $A^{2}$ with a unique radius coverage $r$. Find a set of nodes $M$ with the minimal neighbor density.

We have developed a simulation model developed with Netlogo which is a multi-agent programmable modeling environment. To relax the problem we have introduce a tolerance parameter $\mathrm{p}$ which is the acceptable percentage of nodes declared as border nodes. Figure 6 illustrates simulation results with $\mathrm{N}=3000, \mathrm{~A}=80, \mathrm{p}=\{50 \%, 75 \%\}$ and $r=\{10,20\}$ to test the influence of the coverage in the accuracy. For each combination of coverage radius with tolerance percentage we can see that the quantity of border nodes $\mathrm{M}$ in black color is variable. The mean values of $\mathrm{M}$ after 100 simulations for each pair (coverage radius, tolerance percentage $)$ are: $(10,50 \%)=9.1123 \%,(20,50 \%)=15.2345$ $\%,(10,75 \%)=45.3212 \%,(20,75 \%)=51.3421 \%$.
Therefore, the mean $\mathrm{M}$ value is directly proportional to the pair $(r, p)$.

Another result is the relation between the coverage radius $r$ with the value of the side A. The strategy permits to find border nodes while the value of $r$ is less than $\sqrt{ } 2 \mathrm{~A}$. Beyond this value all the nano nodes within the product will be the same quantity of neighbors. For this, the best strategy to find border nodes is: with high density, low values in the pair (r, $\mathrm{p}$ ), and with low density, high values in the pair (r, p), with $\mathrm{r}<\sqrt{ } 2 \mathrm{~A}$.

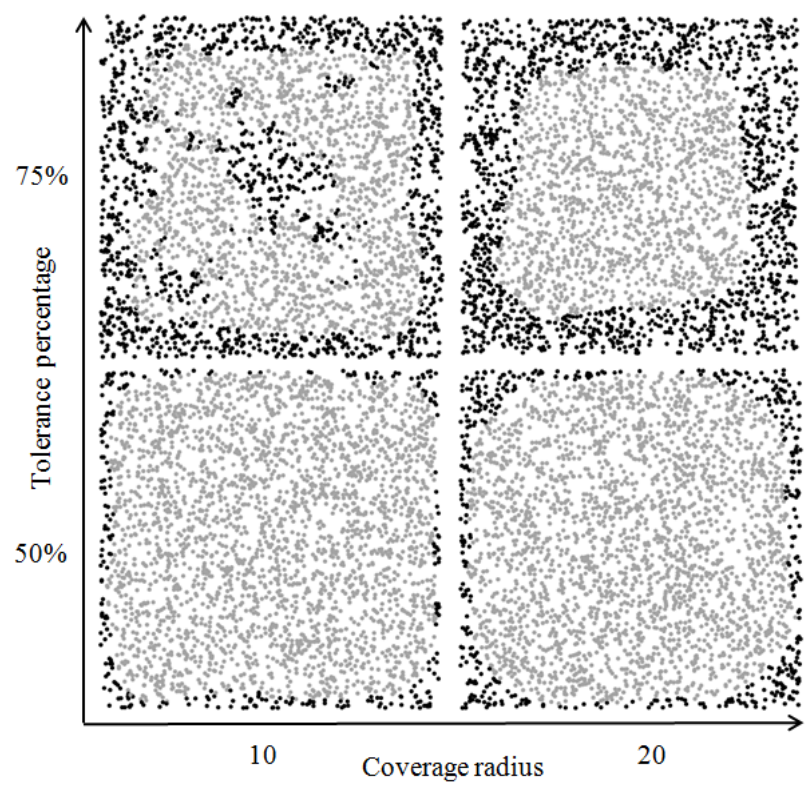

Fig. 6. Border nano nodes: simulation results.

\subsection{Distance estimation}

The previous stage determines the nodes in the product border, i.e. each node is capable to verify itself if it's a border node or not. The next step consists of distance estimation between border nano nodes in order to obtain the dimensions of the product. To ensure autonomy of the PHM systems, each node must be able to execute a localisation technique in order to determine point-to-point distance with others border nodes. Among decentralized ranging techniques, range-based methods are to find the location of the target node using absolute point-to-point distance estimates or angle estimates. techniques involve many techniques such as Time of Arrival (ToA), Angle of Arrival (AoA), Time Difference of Arrival (TDoA) or received signal strength (RSS). The latter presents advantages to be less complex and not require synchronization and clock precision. However RSS requires precise channel behavioral model and is sensitive to channel inconsistency.

The figure 7 shows distance estimation between the border nodes for planar surfaces without material disruptions. The ideal scenario is represented on figure 7.a with an uniform grid distribution of nodes in the product. Direct ranging between these nodes can be perform according to the 
coverage radius. When the border node estimation is less accurate (Fig. 7.b), the process is more complex requiring to realize mutiple measurement or combine techniques. Thus the accuracy obtained at the first step of the strategy is very important.

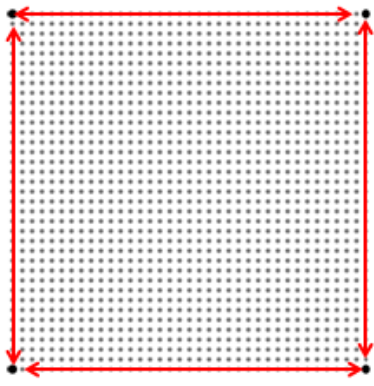

(a)

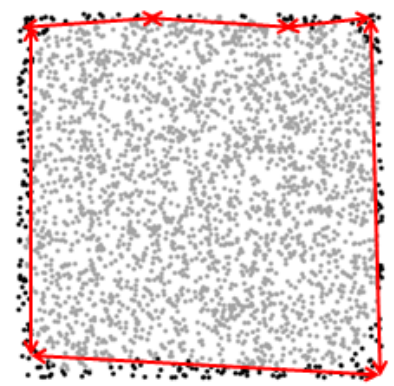

(b)
Fig. 7. Ranging techniques for distance estimation.

Like for WSN, performance studies of localization techniques in WNSN is an open research problem with more constraints related to signal propagation into materials and nano-nodes integration.

\section{CONCLUSIONS}

This paper deals with the development of Health Monitoring systems for products. Based on development of nanotechnologies, and more particular of Wireless Nano Sensor Networks, such a system is consistent with the concept of intelligent product. It offers new monitoring opportunities such as the monitoring throughout product lifecycle. To illustrate this, we have proposed a monitoring strategy of dimensional features of the product. This PHM system, called self-measurement system, provides to the product the ability to determine its own dimensions, even after transformation. To achieve this objective, neighborhood density estimation and distance estimation algorithms are used. This method presents the advantages to use only networked nano-devices with a nano-transceiver.

Although these algorithms could be tested with traditional wireless sensor networks, they are certainly not well suited to the specific features (e.g. network coverage area limitation, low memory and processing abilities) and application requirements of wireless nano sensor networks. Future work involves further analysis of communication in nano networks to develop or adapt techniques of neighborhood discovery and ranging.

\section{REFERENCES}

Akyildiz, I.F. and Jornet J.M. (2010). Electromagnetic wireless nanosensor networks, Nano Communications Networks Journal, vol. 1, no. 1, pp. 3-19.

Atakan, B. and Akan, O.B. (2009). Carbon nanotube sensor networks, Proc. Of IEEE Nanocom, San Francisco.
Balageas, D., Fritzen, C.-P. and Güemes, A. (2010). Structural Health Monitoring, ISTE, London, UK, ISBN: 9780470612071.

Delaney, K. (2008). Ambient Intelligence with Microsystems: Augmented Materials and Smart Objects, SpringerVerlag New York, ISBN: 1441942777.

Främling, K. and McFarlane, D. (2009). Editorial for Special Issue on Intelligent Products. Computers in Industry, Volume 60, Issue 3 April 2009. pp. 135-136.

Kocaoglu M. and Akan O.B. (2012). Minimum energy coding for wireless nanosensor networks. IEEE INFOCOM, page 2826-2830.

Koksal, C.E., Ekici, E. (2010). A nanoradio architecture for interacting nanonetworking tasks, Nano Communications Networks Journal, vol. 1, pp. 63-75.

Legg, S., and Hutter, M. (2007). A collection of definitions of intelligence. Advances in Artificial General Intelligence: Concepts, Architectures and Algorithms (pp. 17-24). Amsterdam, NL: IOS Press.

Meyer, G.G., Främling, K. and Holmström, J. (2009). Intelligent products: A survey. Computers in Industry, 60, 137-148.

Mishra, S., Pecht, M., and Goodman, D. (2002). In-situ Sensors for Product Reliability Monitoring, Proceedings of SPIE, Vol. 4755, pp. 10-19.

Moore, M.J., Nakano, T., Enomoto, A. and Suda, T. (2010). Measuring Distance with Molecular Communication Feedback Protocols, 5th ACM/ICST Int. Conf. BioInspired Models of Network, Information, and Computing Systems.

Niu, G., Singh, S., Holland, S.W. and Petch, M. (2011). Health monitoring of electronic products based on Mahalanobis distance and Weibull decision metrics. Microelectronics Reliability, 51(2):279-284.

Parcerisa, L., Akyildiz, I.F. (2009). Molecular communication options for long range nanonetworks, Computer Networks Journal, 53 (16): 2753-2766.

Robertazzi, T. and Sarachik, P. (1986). Self-organizing communication networks, IEEE Communications Magazine, Vol. 24(1): 28 - 33.

Ventä, O. (2007). Intelligent products and systems. Technical report, VTT.

Vichare, N., Rodgers, P., Azarian, M. and Pecht, M. (2004). Application of Health Monitoring to Product Take-back Decisions, Proceedings of the Joint International Congress and Exhibition - Electronics Goes Green, pp. 945-951, Berlin, Germany. 